\title{
On reduced models in nonlinear solid mechanics
}

\author{
Pierre Ladevèze \\ LMT (ENS Cachan, CNRS, Université Paris-Saclay), 61, avenue du Président Wilson, F-94235 Cachan Cedex, France
}

The capabilities and limits of current model reduction methods are examined in the case of solid mechanics problems involving significant nonlinearities-such as (visco)plasticity, damage, contact with friction, ...-and parameters. Particular emphasis will be put on the PGD method (Proper Generalized Decomposition) and its last developments. These reduced models are the key to the introduction of materials physics in simulation-driven structural design, a domain in which quasi real-time simulations are mandatory.

\section{Introduction}

\subsection{Our main motivation}

Our work is part of the "composites" revolution which the aeronautical industry has engaged in, particularly in Europe. The share of composites in civilian planes has grown a lot and vital elements are made of carbon-fiber laminated composites. The current design approach is prohibitively expensive in cost and duration (and, therefore, today inadequate) because it is based almost exclusively on testing. Today, industrializing a new matrix grade requires carrying out the whole series of tests with their numerous stacking sequences all over again, which inhibits innovation.

This is leading the aeronautical industry to a reversal of the situation through "virtual testing", which consists in replacing, whenever possible, the numerous experimental tests used today by virtual charts (see Fig. 1). This should lead to a significant decrease in the cost and duration of the design and sizing stage. Virtual Charts are particular Reduced Order Models (ROM) associated to goal-oriented quantities. Fig. 2 gives an illustration of a virtual chart for a coupon family of composite plates with a hole, the parameters being the hole diameter as well as the angles defining the fiber directions of the different plies. The virtual chart here gives the maximum of the longitudinal strain in term of the parameters over the parameter set.

E-mail address: ladeveze@lmt.ens-cachan.fr.

1 EUROMECH Solid Mechanics Prize lecture, Madrid 2015.

\subsection{The scope of the paper and the state-of-the-art}

This paper deals with the computation offline of a reducedorder model (ROM) in nonlinear solid mechanics. We focus on complex constitutive relations as (visco)plasticity and damage and also unilateral contact with friction and damage. However, we limit ourselves to quasi-static and small displacements conditions. Loading and material parameters could be stochastic and one considers here that they belong to a given set.

We have been working on ROM computation for 30 years with the so-called LATIN-PGD and what we are doing at the present time is the result of many works. PGD means "Proper Generalized Decomposition" and LATIN denotes the computational method which is nonincremental. The LATIN-PGD method was introduced in Ladevèze et al. (Ladevèze, 1985a, 1985b) for viscoplastic materials whose constitutive relations are described using a functional approach. Its extension to modern material descriptions involving internal variables, still for viscoplastic materials, was proposed in Ladevèze et al. (Ladevèze, 1989; Ladevèze, 1991). A number of mathematical properties regarding convergence and error indicators were proved in the book (Ladevèze, 1999). Overview could be found in Ladevèze et al. (Ladevèze, 1999; Chinesta and Ladevèze, 2014; Ladevèze et al., 2009). Originally, PGD was called radial loading approximation, which, to us, meant a "mechanics" approximation in solid mechanics. In 2010, together with F. Chinesta, we changed the name because PGD can be viewed as an extension of the classical Proper Orthogonal Decomposition (POD).

This paper should be seen as a revisit of the LATIN-PGD leading to a new, general and robust PGD computation technique. This is 

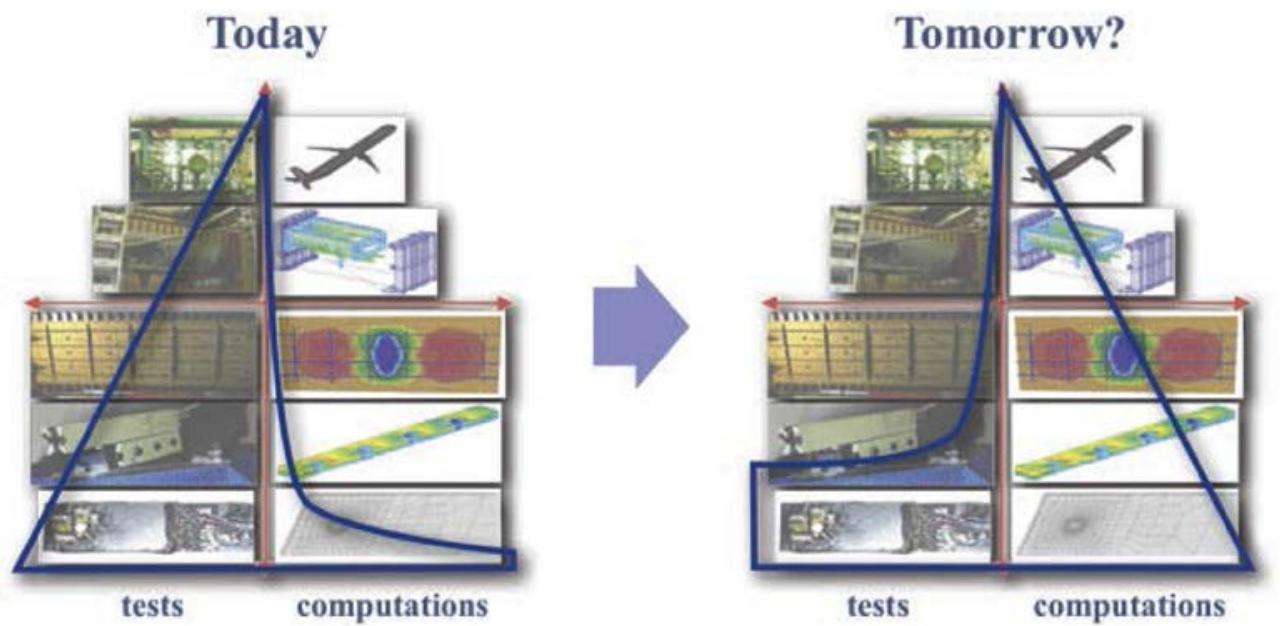

Fig. 1. Future of the test/computation pyramid.
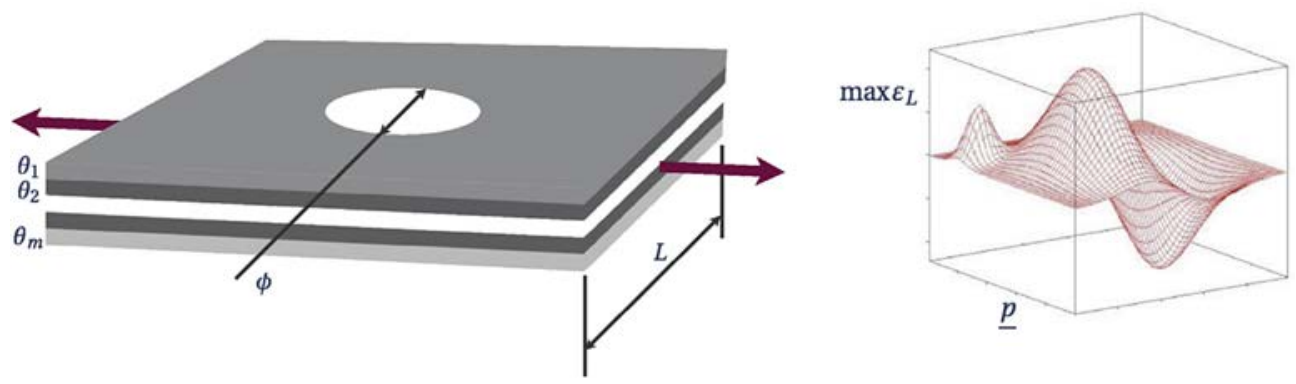

Fig. 2. Virtual chart for a composite coupon family.

based on an "abstract" reformulation of parametric nonlinear solid mechanics problems defined over a time-space domain. This work is the follow-up to (Ladevèze et al., 2010; Relun et al., 2013; Allier et al., 2015; Jessus et al., 2016). Today, there are few other works except the works of Ryckelynck and his group done with POD (Ryckelynck, 2005, 2009; Ryckelynck et al., 2012). However, things are changing and today there are more and more POD-approaches developed in relation with the homogenization technique $\mathrm{FE}^{2}$ (Lamari et al., 2010; Ammar et al., 2007; Hernández et al., 2014; Radermacher and Reese, 2015; Yvonnet et al., 2013). Another attempt has been done recently with the Asymptotic Numerical Method (ANM) but this approach seems to be quite limited. PGD, like any ROM technique for nonlinear problems, can lead to the computation of numerous nonlinear integrals, which can become very expensive. Additional reduction or interpolation are then introduced to reduce their computation cost. This is done offline with the PGD computation. Our answer is the so-called Reference Point Method (RPM), which is recalled here; furthermore, a performance analysis is given in term of the RPM-parameters.

\subsection{Basic features of the new generation of ROM computational methods}

By new generation of ROM computational methods, we intend Reduced Based method (RB) (Patera and Rozza, 2006; Rozza and Veroy, 2007; Maday and Ronquist, 2004; Barrault et al., 2004), POD (Kunisch and Xie, 2005; Lieu et al., 2006; Gunzburger et al., 2007) and PGD methods. They are based on the same ideas. The first and main idea is that the shape functions are not as usual a priori given. They are computed simultaneously with the solution itself thanks to an iterative procedure. For a problem defined over the time-space domain, the solution is then written as:

$\mathbf{s}(t, \boldsymbol{x}) \approx \mathbf{s}^{m}(t, \boldsymbol{x})=\sum_{i=1}^{m} a_{i} \Psi_{i}(t, \boldsymbol{x})$

The second idea is to introduce a variable separation hypothesis or something equivalent:

$\boldsymbol{\Psi}_{i}(t, \boldsymbol{x})=\lambda_{i}(t) \boldsymbol{\Gamma}_{i}(\boldsymbol{x})$

where the time functions $\lambda_{i}$ and the space functions $\boldsymbol{\Gamma}_{i}$ are arbitrary. That is a departure from other approximation methods for which shape functions are a priori given or partially given. However, ROM computational techniques are quite different (Chinesta and Ladevèze, 2014).

The PGD is characterized by two ingredients: the variable separation hypothesis and a residual which should be minimized or something equivalent. Let $R$ be the residual which satisfies:

$\forall \mathbf{s} \in \mathbf{S}^{[0, T]}, R(\mathbf{s}) \geqslant 0 \quad$ and $\quad R(\mathbf{s})=0 \Rightarrow \mathbf{S}=\mathbf{s}_{e x}$

the problem to solve is then:

$\min _{\mathbf{s}^{m} \in \mathbf{S}^{[0, T]}} R\left(\mathbf{s}^{m}\right)$

which is twice nonlinear. Mechanically, it is nonlinear. Furthermore, the computation of a PGD approximation is always a nonlinear problem. 
Remark. - The simplest case is the situation where one computes the PGD of a given function. It has been proved that the time functions are the eigenfunctions of a certain eigenvalue problem (Ladevèze, 1999). For the $L^{2}$ norm, PGD is exactly the classical Singular Value Decomposition (Golub and Van Loan, 1996). It follows that PGD modes can be seen as "eigenmodes".

\section{The reference problem}

With the assumption of small perturbations, let us consider the quasi-static and isothermal evolution of a structure defined over the time-space domain $[0, T] \times \Omega$. This structure is subjected to prescribed body forces $\boldsymbol{f}_{d}$, traction forces $\boldsymbol{F}_{d}$ over a part $\partial_{2} \Omega$ of the boundary, and displacements $\boldsymbol{u}_{d}$ over the complementary part $\partial_{1} \Omega$ (see Fig. 3).

The state of the structure is defined by the set of the fields $\mathbf{s}=\left(\dot{\boldsymbol{\varepsilon}}_{\mathrm{p}}, \dot{\boldsymbol{X}}, \boldsymbol{\sigma}, \boldsymbol{Y}\right)$ (where the dot notation $\dot{\square}$ denotes the time derivative), in which:

- $\varepsilon_{\mathrm{p}}$ designates the inelastic part of the strain field $\boldsymbol{\varepsilon}$ which corresponds to the displacement field $\boldsymbol{u}$, uncoupled into an elastic part $\boldsymbol{\varepsilon}_{\mathrm{e}}$ and an inelastic part $\boldsymbol{\varepsilon}_{\mathrm{p}}=\boldsymbol{\varepsilon}-\boldsymbol{\varepsilon}_{\mathrm{e}} ; \boldsymbol{X}$ designates the remaining internal variables;

- $\boldsymbol{\sigma}$ designates the Cauchy stress field and $\boldsymbol{Y}$ the set of variables conjugate of $\boldsymbol{X}$ ( $\boldsymbol{Y}$ and $\boldsymbol{X}$ have the same dimension). $\boldsymbol{X}$ could be hardening variables, damage variables, chemical variables, ...

All these quantities are defined over the time-space domain $[0, T] \times \Omega$ and assumed to be sufficiently regular. For the sake of simplicity, the displacement $\boldsymbol{u}$ is assumed to have a null initial value.

Introducing the following notations for the primal fields:

$\mathbf{e}_{\mathrm{p}}=\left[\begin{array}{c}\boldsymbol{\varepsilon}_{\mathrm{p}} \\ -\boldsymbol{X}\end{array}\right], \mathbf{e}=\left[\begin{array}{c}\boldsymbol{\varepsilon} \\ 0\end{array}\right]$ and $\mathbf{e}_{\mathrm{e}}=\left[\begin{array}{c}\boldsymbol{\varepsilon}_{\mathrm{e}} \\ \boldsymbol{X}\end{array}\right]$ so that $\mathbf{e}_{\mathrm{p}}=\mathbf{e}-\mathbf{e}_{\mathrm{e}}$

and for the dual fields:

$\mathbf{f}=\left[\begin{array}{l}\boldsymbol{\sigma} \\ \boldsymbol{Y}\end{array}\right]$

The mechanical dissipation rate for the entire structure $\Omega$ is:

$\int_{\Omega}\left(\dot{\boldsymbol{\varepsilon}}_{\mathrm{p}}: \boldsymbol{\sigma}-\dot{\boldsymbol{X}} \cdot \boldsymbol{Y}\right) d \Omega=\int_{\Omega}\left(\dot{\mathbf{e}}_{\mathrm{p}} \circ \mathbf{f}\right) d \Omega$

where - denotes the contraction adapted to the tensorial nature of $\boldsymbol{X}$ and $\boldsymbol{Y}$. Notation o denotes the contraction operator for

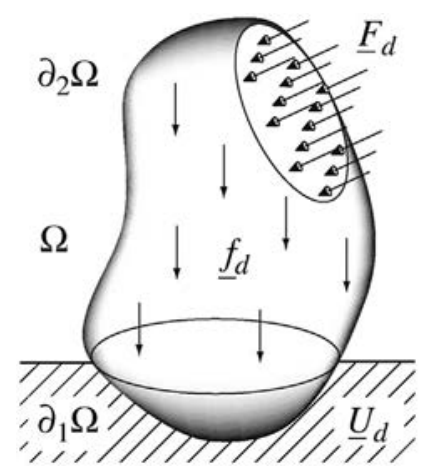

Fig. 3. The reference problem. generalized quantities. Let us introduce the following fundamental bilinear "dissipation" form:

$\left\langle\mathbf{s}, \mathbf{s}^{\prime}\right\rangle=\int_{[0, T] \times \Omega}\left(1-\frac{t}{T}\right)\left(\dot{\mathbf{e}}_{\mathrm{p}} \circ \mathbf{f}^{\prime}+\dot{\mathbf{e}}_{\mathrm{p}}{ }^{\prime} \circ \mathbf{f}\right) d \Omega d t$

along with $\mathbf{E}$ and $\mathbf{F}$, the spaces of the fields $\dot{\mathbf{e}}_{\mathrm{p}}$ and $\mathbf{f}$ which are compatible with (8). These spaces enable us to define $\mathbf{S}=\mathbf{E} \times \mathbf{F}$, the space in which the state $\mathbf{s}=\left(\dot{\mathbf{e}}_{\mathrm{p}}, \mathbf{f}\right)$ of the structure is being sought.

Following (Ladevèze, 1999), a normal formulation with internal state variables is used to represent the behavior of the material. If $\rho$ denotes the mass density of the material, from the free energy $\rho \Psi\left(\boldsymbol{\varepsilon}_{\mathrm{e}}, \boldsymbol{X}\right)$ with the usual uncoupling assumptions, the state law yields:

$\boldsymbol{\sigma}=\rho \frac{\partial \psi}{\partial \boldsymbol{\varepsilon}_{\mathrm{e}}}=\mathbf{K} \boldsymbol{\varepsilon}_{\mathrm{e}}$

$\boldsymbol{Y}=\rho \frac{\partial \psi}{\partial \boldsymbol{X}}=\boldsymbol{\Lambda} \boldsymbol{X}$

where the Hooke's tensor $\mathbf{K}$ and the constant, symmetric and positive definite tensor $\boldsymbol{\Lambda}$ are material characteristics.

The state evolution laws can be written:

$\dot{\mathbf{e}}_{\mathrm{p}}=\mathbf{B}(\mathbf{f}) \quad$ with $\quad \mathbf{e}_{\mathrm{p} \mid t=0}=0$

where $\mathbf{B}$ is a positive operator which is also for most viscoplastic models maximal monotone (Ladevèze, 1999). Let us introduce now the space $\mathscr{U}_{a d}^{[0, T]}$ of admissible displacement fields $\boldsymbol{u}$ defined over $[0, T] \times \Omega$ and $\mathscr{U}_{a d, 0}^{[0, T]}$ the associated vectorial space. The compatibility equation can be written as:

Find $\boldsymbol{u} \in \mathscr{U}_{a d}^{[0, T]}$ such that $\forall \boldsymbol{u}^{\star} \in \mathscr{U}_{a d, 0}^{[0, T]}$

$$
\begin{aligned}
\int_{[0, T] \times \Omega} \operatorname{Tr}\left[\boldsymbol{\varepsilon}(\boldsymbol{u}) \mathbf{K} \boldsymbol{\varepsilon}\left(\boldsymbol{u}^{\star}\right)\right] d \Omega d t= & \int_{[0, T] \times \Omega} \operatorname{Tr}\left[\boldsymbol{\varepsilon}_{\mathrm{p}} \mathbf{K} \boldsymbol{\varepsilon}\left(\boldsymbol{u}^{\star}\right)\right] d \Omega d t \\
& +\int_{[0, T] \times \Omega} \boldsymbol{f}_{d} \cdot \boldsymbol{u}^{\star} d \Omega d t \\
& +\int_{[0, T] \times \partial_{2} \Omega} \boldsymbol{F}_{d} \cdot \boldsymbol{u}^{\star} d S d t
\end{aligned}
$$

It follows that the stress $\boldsymbol{\sigma}=\mathbf{K}\left(\boldsymbol{\varepsilon}(\boldsymbol{u})-\boldsymbol{\varepsilon}_{\mathrm{p}}\right)$ can be written:

$\boldsymbol{\sigma}=\boldsymbol{\Omega} \varepsilon_{\mathrm{p}}+\boldsymbol{r}_{d}$

where $\boldsymbol{\Omega}$ is a linear given operator and $\mathbf{r}_{d}$ is a prestress depending on the data. Introducing the generalized stress, the admissibility conditions can be written as:

$\mathbf{f}=\mathbf{Q} \mathbf{e}_{\mathrm{p}}+\mathbf{r}_{d}$

with

$\mathbf{Q}=\left[\begin{array}{ll}\boldsymbol{\Omega} & \mathbf{0} \\ \mathbf{0} & \boldsymbol{\Lambda}\end{array}\right]$ and $\mathbf{r}_{d}=\left[\begin{array}{c}\mathbf{r}_{d} \\ \mathbf{0}\end{array}\right]$

where $\mathbf{Q}$ is a linear symmetric positive operator. Finally, the problem to solve is:

Find $\mathbf{s}=\left(\dot{\mathbf{e}}_{\mathrm{p}}, \mathbf{f}\right) \in \mathbf{S}^{[0, T]}$ such that:

$\mathbf{f}=\mathbf{Q} \mathbf{e}_{\mathrm{p}}+\mathbf{r}_{d} \quad$ and $\quad \dot{\mathbf{e}}_{\mathrm{p}}=\mathbf{B}(\mathbf{f}) \quad$ with $\quad \mathbf{e}_{\mathrm{p} \mid t=0}=0$

Consequently, one has to solve a first order differential equation with an initial condition; The operators $\mathbf{Q}$ and $\mathbf{B}$ as well as the right- 
hand-side member $\mathbf{r}_{d}$ could depend on the parameter $\mu$ belonging to the parameter set $\boldsymbol{\Sigma}_{\mu}$.

\section{The solver LATIN for ROM computation}

\subsection{ROM in nonlinear solid mechanics}

Let us consider ROM computations based on time/space separation. A natural and general idea is to transform the reference problem into a succession of linear global problems over $[0, T] \times \Omega$ which could depend on parameters. Using RB, POD or PGD, a Reduced Order Model can be built over $[0, T] \times \Omega$ for each linear problem. The final ROM is then obtained gathering all the previous ROM.

\subsection{Our answer: the solver LATIN}

The LATIN method is an iterative strategy which differs from classical incremental or step-by-step techniques in that, at each iteration, it produces an approximation of the complete structural response over the whole loading history being considered. In other words, the name LATIN for Large Time Increment method was not chosen very well because the method is essentially nonincremental. A review of the state-of-the-art and more recent extensions could be found in Ladevèze et al. (Ladevèze, 1999; Chinesta and Ladevèze, 2014; Ladevèze et al., 2009; Ladevèze and Perego, 2000).

The LATIN method is designed as a mechanics-based computational strategy whose aim is to achieve the best possible performance level for solid mechanics problems. Consequently, this alternative approach is rooted in some remarkable properties which are verified by most of the models encountered in structural mechanics.

The LATIN method operates over the time-space domain $[0, T] \times \Omega$, and its first principle (P1) consists in separating the difficulties. Thus, the equations are divided into (see Fig. 4):

- a set of linear equations which can be global in the space variables: the equilibrium and compatibility equations, the state equations;

- a set of equations which are local in the space variables but can be nonlinear: the state evolution laws.

The reformulation (15) of the reference problem enters into this framework because:

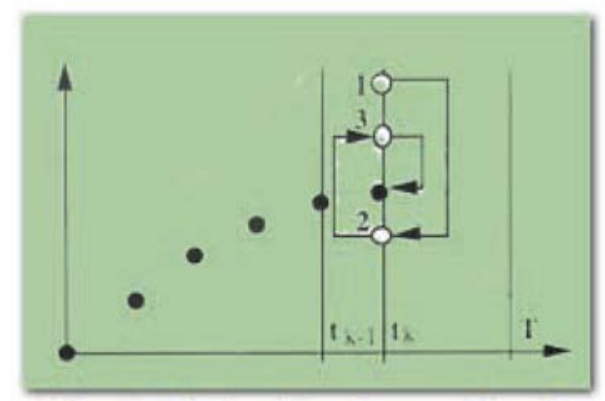

Classical step-by-step methods
- $\mathbf{Q}$ is a linear operator;

- $\mathbf{B}$ is at least local in space variables.

In the geometric representation given Fig. $5, \mathbf{A}_{\mathbf{d}}$ and $\boldsymbol{\Gamma}$ represent the solutions of the first and second set respectively. The exact solution is the intersection of $\mathbf{A}_{\mathbf{d}}$ and $\boldsymbol{\Gamma}$. The partition into $\mathbf{A}_{\mathbf{d}}$ and $\boldsymbol{\Gamma}$ is very natural, $\mathbf{A}_{\mathbf{d}}$ being associated to the free energy and $\boldsymbol{\Gamma}$ with the dissipation. The LATIN second principle (P2) is also very natural. It consists in solving the two sets of equations alternatively until practical convergence. In order to do that, one uses search directions given as parameters. One possible choice (Newton search direction) consists of the tangent direction and its conjugate direction (see Fig. 6).

Local stage at iteration $n+1 .-$ Find $\widehat{\mathbf{s}}_{n+1 / 2}=\left(\widehat{\dot{\mathbf{e}}}_{\mathrm{p}, n+1 / 2}, \widehat{\mathbf{f}}_{n+1 / 2}\right) \in \mathbf{S}^{[0, T]}$ such that:

$$
\begin{aligned}
& \widehat{\dot{\mathbf{e}}}_{\mathrm{p}, n+1 / 2}=\mathbf{B}\left(\widehat{\mathbf{f}}_{n+1 / 2}\right) \quad \text { with } \quad \widehat{\mathbf{e}}_{\mathrm{p}, n+1 / 2}=0 \text { at } t=0 \\
& \widehat{\dot{\mathbf{e}}}_{\mathrm{p}, n+1 / 2}-\dot{\mathbf{e}}_{\mathrm{p}, n}+\mathbf{H}^{+}\left(\widehat{\mathbf{f}}_{n+1 / 2}-\mathbf{f}_{n}\right)=0
\end{aligned}
$$

The search direction $\mathbf{H}^{+}$is a parameter. Practically, one takes a linear positive operator which is local both on time and space variables. It follows that the problem to solve is local on the space variable and then can be split into small independent problems associated to Gauss points. This local stage is very suitable for parallel computing.

Linear stage at iteration $n+1$. - Find $\mathbf{s}_{n+1}=\left(\dot{\mathbf{e}}_{\mathrm{p}, n+1}, \mathbf{f}_{n+1}\right) \in \mathbf{S}^{[0, T]}$ such that:

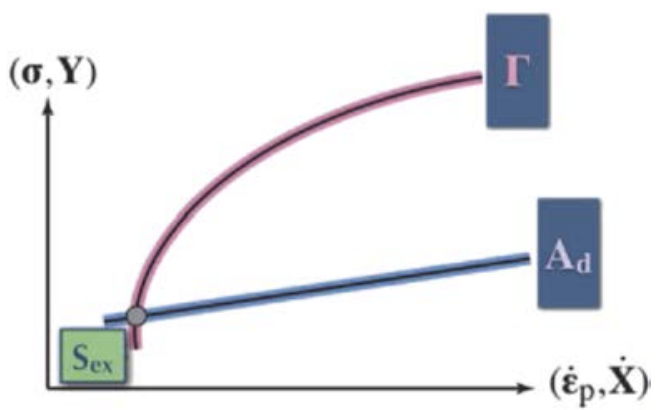

Fig. 5. The geometric representation associated to the reformulation of the reference problem.

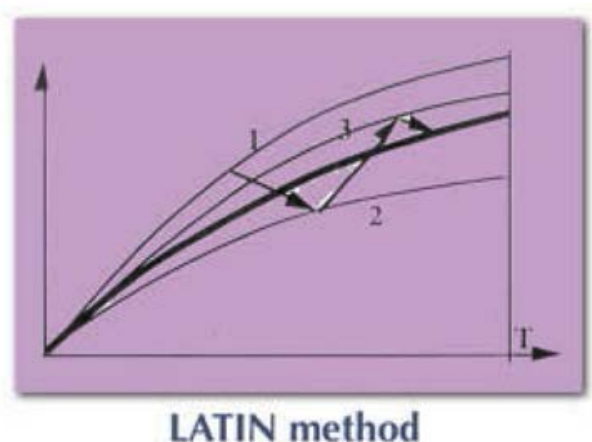

LATIN method

Idea: performance $カ \rtimes=$ mechanics-based

Fig. 4. The LATIN method and classical step-by-step methods. 


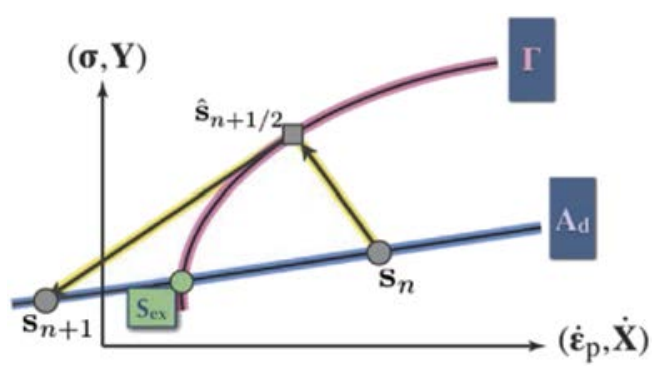

Fig. 6. Iteration $n+1$ of the LATIN method over $[0, T] \times \Omega$.

$\mathbf{f}_{n+1}=\mathbf{Q} \mathbf{e}_{\mathrm{p}, n+1}+\mathbf{r}_{d}$
$\dot{\mathbf{e}}_{\mathrm{p}, n+1}-\widehat{\dot{\mathbf{e}}}_{\mathrm{p}, n+1 / 2}-\mathbf{H}^{-}\left(\mathbf{f}_{n+1}-\widehat{\mathbf{f}}_{n+1 / 2}\right)=0$ with $\mathbf{e}_{\mathrm{p}, n+1}=0$ at $t=0$

The search direction $\mathbf{H}^{-}$is a parameter. This is a linear positive operator which is local both on time and space variables. It is associated to the material operator $\mathbf{B}$. One has to solve a first order linear differential equation with an initial condition, the operator $\mathbf{Q}$ being non-explicit.

Remark. - In practice, $\mathbf{H}^{-}$is chosen close to the tangent to the manifold $\boldsymbol{\Gamma}$ at the point $\widehat{\mathbf{s}}_{n+1 / 2}=\left(\widehat{\dot{\mathbf{e}}}_{\mathrm{p}, n+1 / 2}, \widehat{\mathbf{f}}_{n+1 / 2}\right)$. For $\mathbf{H}^{+}$, one takes $\mathbf{0}$ or $\mathbf{H}^{-}$.

Convergence of the LATIN solver. - The convergence of the iterative process has been proved in the case of non-softening materials and contacts without friction (Ladevèze, 1999). Precisely, the iterative process converges if:

- the material operator $\mathbf{B}$ is maximal monotone;

- the material operator $\boldsymbol{\Lambda}$ is positive definite;

- the search directions $\mathbf{H}^{-}$and $\mathbf{H}^{+}$are positive definite and equal $\mathbf{H}^{-}=\mathbf{H}^{-}$.

The distance between two successive approximations gives a good and easily computed error indicator. Let us also note that one often uses an additional relaxation with a coefficient equal to 0.8 .

\section{PGD computation}

\subsection{The linear stage at iteration $n+1$}

Let us introduce corrections:

$\Delta \dot{\mathbf{e}}_{\mathrm{p}}=\dot{\mathbf{e}}_{\mathrm{p}, n+1}-\dot{\mathbf{e}}_{\mathrm{p}, n}$
$\Delta \mathbf{f}=\mathbf{f}_{n+1}-\mathbf{f}_{n}$

where $\mathbf{s}_{n+1}=\left(\dot{\mathbf{e}}_{\mathrm{p}, n}, \mathbf{f}_{n}\right)$ has been computed at iteration $n$. The problem to solve over $[0, T] \times \Omega$ at iteration $n+1$ is then:

Find $\Delta \mathbf{s}=\left(\Delta \dot{\mathbf{e}}_{\mathrm{p}}, \Delta \mathbf{f}\right) \in \mathbf{S}^{[0, T]}$ such that

$\Delta \mathbf{f}=\mathbf{Q} \Delta \dot{\mathbf{e}}_{\mathrm{p}}$

$\Delta \dot{\mathbf{e}}_{\mathrm{p}}-\mathbf{H}^{-} \Delta \mathbf{f}=\mathbf{R}_{d} \quad$ with $\quad \Delta \mathbf{e}_{\mathrm{p}}=0$ at $t=0$

\subsection{The PGD computational tools}

Numerous constitutive relations have been studied: viscoplasticity, damage, large displacements and deformations, contacts with friction. Various Galerkin computational approaches have been considered in order to increase robustness. Surveys can be founded in Ladevèze et al. (Ladevèze, 1999; Chinesta and Ladevèze,
2014; Ladevèze et al., 2009). However, it is only recently that a new way leading to a truly robust version has been introduced and developed. It is a non-Galerkin approach described in Ladevèze et al. (Ladevèze, 2008; Allier et al., 2015; Jessus et al., 2016). This robust PGD computation method is extended here to a large family of constitutive relations.

\subsection{A robust PGD computation method}

\subsubsection{The residual}

Let us consider the problem (19) where the second equation is related to the search direction. The main idea is to interpret it as a linear constitutive relation, the operator $\mathbf{H}^{-}$being local both on time and space variables and positive definite as the Hooke tensor. Consequently, one introduces the associated constitutive relation error which will be minimized:

$$
\begin{aligned}
r(\Delta \mathbf{s}, t)= & \frac{1}{2} \int_{\Omega}\left[\Delta \dot{\mathbf{e}}_{\mathrm{p}}-\mathbf{H}^{-} \Delta \mathbf{f}-\mathbf{R}_{d}\right]\left(\mathbf{H}^{-}\right)^{-1}\left[\Delta \dot{\mathbf{e}}_{\mathrm{p}}-\mathbf{H}^{-} \Delta \mathbf{f}\right. \\
& \left.-\mathbf{R}_{d}\right] d \Omega
\end{aligned}
$$

and

$R(\Delta \mathbf{s})=\int_{[0, T]}\left(1-\frac{t}{T}\right) r(\Delta \mathbf{s}, t) d t$

with $\Delta \mathbf{S}=\left(\Delta \dot{\mathbf{e}}_{\mathrm{p}}, \Delta \mathbf{f}\right) \in \mathbf{S}^{[0, T]}$. The problem (19) becomes:

Find $\Delta \mathbf{s} \in \mathbf{S}^{[0, T]}$ minimizing

$\Delta \mathbf{s} \in \mathbf{S}^{[0, T]} \mapsto R(\mathbf{s}) \in \mathbb{R}$

with the constrains $\Delta \mathbf{f}=\mathbf{Q} \Delta \dot{\mathbf{e}}_{\mathrm{p}}$ and $\quad \Delta \mathbf{e}_{\mathrm{p}}=0$ at $t=0$

Remark. - The time-residual $r(\Delta \mathbf{s}, t)$ can be used to build ROM with RB method.

\subsubsection{Time/space variable separation hypothesis}

One only prescribes that:

$\Delta \mathbf{e}_{\mathrm{p}}=\sum_{i=1}^{m} \lambda_{i}(t) \mathbf{g}_{i}(\boldsymbol{x})$

with $\lambda_{i}(0)=0$ (initial condition), $\mathbf{g}_{i} \in L^{2}(\Omega)$ and $\lambda_{i}(t) \in L^{2}[0, T]$. It follows, using admissibility conditions, that:

$\Delta \mathbf{f}=\sum_{i=1}^{m} \lambda_{i}(t) \mathbf{Q g}_{i}(\boldsymbol{x})$

where $\mathbf{Q g}_{i}(\boldsymbol{x})$ are computed solving several elasticity problems.

\subsubsection{Minimization technique}

Let us start with:

$\mathbf{s}_{n}=\left(\dot{\mathbf{e}}_{\mathrm{p}}^{0}, \mathbf{f}^{0}\right)+\sum_{i=1}^{m}\left(\dot{\lambda}_{i} \mathbf{E}_{\mathrm{p}}^{i}, \lambda_{i} \mathbf{F}^{i}\right)$

The iteration $n+1$ has two steps.

Step 1: Updating of the PGD time-functions. - This POD phase relies on the space PGD modes $\left(\mathbf{E}_{\mathrm{p}}^{i}, \mathbf{F}^{i}\right)$ for which the computation cost is relatively high. New time-functions, still noted $\lambda_{i}$ are computed minimizing the residual $R$ with the constraint $\lambda_{i}(0)=0$. One gets a small system of differential equations over the time interval with conditions at the two extremities. The problem can be also solved globally over the time interval $[0, T]$. 
Step 2: Addition of a new PGD mode. - One computes following a "greedy" algorithm:

$\mathbf{s}_{n+1}=\mathbf{s}_{n}+\left(\dot{\lambda} \mathbf{E}_{\mathrm{p}}, \lambda \mathbf{F}\right)$

with $\lambda(0)=0$. The additional PGD mode is obtained through the minimization of the residual $R$, alternatively on the time function $\lambda$ and on the space function $\mathbf{E}_{\mathrm{p}}$. The initialisation of this iterative process is done taking as the first time function guess the root square of the time residual $r(\mathbf{0}, t)$. The minimization with respect to the space variables leads to the resolution of a time-independent spatial problem defined over $\Omega$; that is a classical FE problem. The minimization with respect to the time variable leads to a scalar differential equation over $[0, T]$ with conditions at the two extremities whose resolution is quite inexpensive; the easier way is to solve the global time-problem coming from the residual minimization. The iterative process is stopped after few iterations, practically 2 or 3 . Let us also note that this second step is cancelled if the residual $R(\mathbf{0})$ is relatively small.

\subsubsection{Some remarks}

Number of computed PGD modes. - For most nonlinear problems we computed, the number of needed computed PGD modes does not exceed 20.

Optimality. - Here, one compares for a given accuracy the number of PGD modes computed with the LATIN-PGD with the number of PGD modes computed directly from the exact solution. For transient linear diffusion problems, we have proved numerically that this PGD computational method is quasi-optimal (Allier et al., 2015).

Low-cost version. - The dimension of the space problem to be solved in the PGD computational method described previously is twice the usual one. A low cost version has been derived modifying the computation technique of the space PGD modes; they are computed using the classical Galerkin approach associated to the problem reformulation (24) and then the dimension of the spatial PGD problem is the usual one. In Allier et al. (Allier et al., 2015), the two approaches are compared still on a transient linear diffusion problem: for a given accuracy, the low cost version is much more suitable in terms of cost even if one needs to compute more PGD modes. Numerical illustrations given here after are computed based on to the low cost version. To be clear, let us introduce the Galerkin computational approach of the space PGD modes and then of:

$\Delta \mathbf{s}=\left(\dot{\lambda} \mathbf{E}_{\mathrm{p}}, \lambda \mathbf{F}\right)$

for a given $\lambda$. One supposes for the sake of simplicity, that the search direction $\mathbf{H}^{-}$is diagonal per block, that is:

$\mathbf{H}^{-} \mathbf{f}=\left[\begin{array}{ll}\mathbf{H}_{11}^{-} \boldsymbol{\sigma} \\ \mathbf{H}_{22}^{-} \boldsymbol{Y}\end{array}\right]$

One has from (19):

$$
\begin{aligned}
& \int_{[0, T]}\left(1-\frac{t}{T}\right) \int_{\Omega}\left[\Delta \dot{\boldsymbol{\varepsilon}}_{\mathrm{p}}-\mathbf{H}_{11}^{-} \Delta \boldsymbol{\sigma}-\boldsymbol{\sigma}_{d}\right] \boldsymbol{\sigma}^{\star} d \Omega d t \\
& \quad=0, \quad \forall \boldsymbol{\sigma}^{\star} \in \mathscr{S}_{a d, 0}^{[0, T]}
\end{aligned}
$$

where $\mathscr{S}_{a d, 0}^{[0, T]}$ is the space of the stress fields satisfying equilibrium equations over $[0, T] \times \Omega$ for null loadings. Introducing $\Delta \mathbf{s}$ i.e. $\Delta \dot{\boldsymbol{\varepsilon}}_{\mathrm{p}}=$ $\dot{\lambda} \Sigma_{\mathrm{p}}$ and $\Delta \boldsymbol{\sigma}=\lambda \boldsymbol{C}$, one gets:

$$
\begin{aligned}
& \int_{[0, T]}\left(1-\frac{t}{T}\right) \int_{\Omega}\left[\dot{\lambda} \lambda \boldsymbol{\Sigma}_{\mathrm{p}}-\mathbf{H}_{11}^{-} \lambda^{2} \boldsymbol{C}-\lambda \boldsymbol{\sigma}_{d}\right] \boldsymbol{C}^{\star} d \Omega d t \\
& \quad=0, \quad \forall \boldsymbol{C}^{\star} \in \mathscr{S}_{a d, 0}^{[0, T]}
\end{aligned}
$$

Let us note that $\boldsymbol{\varepsilon}=\boldsymbol{\varepsilon}_{\mathrm{p}}+\mathbf{K}^{-1} \boldsymbol{\sigma}$ leads to:

$$
\int_{\Omega}\left[(\dot{\lambda} \lambda) \mathbf{K}^{-1} \boldsymbol{C}+\left(\lambda^{2} \mathbf{H}_{11}^{-}\right) \boldsymbol{C}+\left(\lambda \boldsymbol{\sigma}_{d}\right)\right] \boldsymbol{C}^{\star} d \Omega=0, \quad \forall \boldsymbol{C}^{\star} \in \mathscr{S}_{a d, 0}^{[0, T]}
$$

where $(\bullet)=\int_{[0, T]}\left(1-\frac{t}{T}\right) \bullet d t$. Introducing $\boldsymbol{\Omega C}=(\dot{\lambda} \lambda) \mathbf{K}^{-1} \boldsymbol{C}+\left(\lambda^{2} \mathbf{H}_{11}^{-}\right) \boldsymbol{C}$ where $\boldsymbol{\Omega}$ is a symmetric positive definite linear operator, one obtains from the previous equation:

$\boldsymbol{\Omega C}+\left(\lambda \boldsymbol{\sigma}_{d}\right)=\boldsymbol{\varepsilon}(\tilde{\boldsymbol{u}})$

where $\tilde{\boldsymbol{u}} \in \mathscr{U}_{\text {ad, }, 0}$. Consequently to compute $\boldsymbol{C}$, ones computes $\tilde{\boldsymbol{u}} \in \mathscr{U}_{a d, 0}$ minimizing:

$$
\begin{aligned}
\mathscr{U}_{a d, 0} & \rightarrow \mathbb{R} \\
\tilde{\boldsymbol{u}}^{\prime} & \mapsto \frac{1}{2} \int_{\Omega} \operatorname{Tr}\left[\boldsymbol{\varepsilon}\left(\tilde{\boldsymbol{u}}^{\prime}\right) \boldsymbol{\Omega}^{-1} \boldsymbol{\varepsilon}\left(\tilde{\boldsymbol{u}}^{\prime}\right)\right] d \Omega-\int_{\Omega} \operatorname{Tr}\left[\left(\lambda \boldsymbol{\sigma}_{d}\right) \boldsymbol{\Omega}^{-1} \boldsymbol{\varepsilon}\left(\tilde{\boldsymbol{u}}^{\prime}\right)\right] d \Omega
\end{aligned}
$$

The computation of the additional variables is easier. From (19), one has:

$\Delta \boldsymbol{Y}=\mathbf{\Lambda} \Delta \boldsymbol{X}$

$\Delta \dot{\boldsymbol{X}}=\mathbf{H}_{22}^{-} \Delta \boldsymbol{Y}+\boldsymbol{X}_{d}$

Introducing $\Delta \boldsymbol{X}=\lambda \boldsymbol{\Gamma}, \boldsymbol{\Gamma}$ is defined by:

$\left[(\dot{\lambda} \lambda)+\left(\lambda^{2} \mathbf{H}_{22}^{-}\right) \boldsymbol{\Lambda}\right] \boldsymbol{\Gamma}=\left(\lambda \boldsymbol{X}_{d}\right)$

and it follows: $\Delta \boldsymbol{Y}=\boldsymbol{\Lambda} \Delta \boldsymbol{X}$.

Problems with parameters. - To solve parametrized problems, PGD has been popularized by the works of Chinesta et al. who proposed to introduce parameters as new coordinates in the decomposition (see (Chinesta et al., 2013) for a survey). The problems that are classically considered are linear and concern parameters (material characteristic, width of a structure, loading amplitude ...) that belong to a certain range of variation and then influence continuously the solution. Here we are considering timedependent nonlinear problems for which the operators $\mathbf{Q B}$ and $\mathbf{r}_{d}$ depend on parameters $\mu$ belonging to $\boldsymbol{\Sigma}_{\mu}$. The previous PGD techniques can be extended easily. The two ingredients, the residual and the variable separation hypothesis, are now:

$$
\begin{aligned}
& R(\Delta \mathbf{s})=\int_{\mathbf{\Sigma}_{\mu}} d \boldsymbol{\mu} \int_{[0, T]} r(\Delta \mathbf{s}, t, \boldsymbol{\mu}) d t \\
& \Delta \mathbf{e}_{\mathrm{p}}=\sum_{i=1}^{m} \lambda_{i}(t) \gamma_{i}(\boldsymbol{\mu}) g_{i}(\boldsymbol{x})
\end{aligned}
$$

This technique works well but is intrusive. Then, for parameters less than 10, we prefer to use another technique based on a remarkable property of the LATIN method: the initialisation of the iterative process could be any function defined over $[0, T] \times \Omega$. The technique is very simple. We cover step-by-step the parameter set; for a new set of parameters, one starts with an initialisation which is the solution obtained for the previous parameter set (Boucard 
and Ladevèze, 1999; Heyberger et al., 2013). This technique is used here with the low cost PGD computational technique. Recently, we have proposed in (Courard et al., 2016) an extension to "positional parameters" which corresponds to situations that do not arise from a classical discretization and can be totally disconnected (different loading cases, different types of material ...).

\section{Additional "reduction" or interpolation}

\subsection{The cost problem and classical answers}

Let us note that the LATIN-PGD needs to compute numerous integrals as:

$I=\int_{[0, T]} \int_{\bigcup} f(t, \boldsymbol{x}) \mathbf{H}(t, \boldsymbol{x}) g(t, \boldsymbol{x}) d \bigcup d t$

where $\mathbf{H}$ changes along the iterations. It can be time-dependent and also nonlinear in term of the computed solution. $f$ and $g$ are not necessarily represented in the PGD framework. It follows that the computation of $I$ requires to loop on all the time steps and all the space Gauss points and consequently its cost could be high. For ROM computations where such integral computation are done online, this problem is a crucial one. This is not the case of the LATIN-PGD where all these calculations are done offline; however, it is always interesting to reduce the computation cost. Several additional reduction or interpolation methods have been proposed to overcome this difficulty. A very popular technique is the Empirical Interpolation Method (EIM) (Barrault et al., 2004; Grepl et al., 2007) and its discrete version named DEIM (Chaturantabut and Sorensen, 2010). The Hyperreduction method (Ryckelynck, 2005, 2009; Ryckelynck et al., 2012) makes the most of a restricted subdomain of the space domain. Other techniques can be seen as particular Gappy-POD methods (Everson and Sirovich, 1994; Astrid et al., 2008; Carlberg and Farhat, 2010). In the next paragraph is detailed the Reference Point Method (RPM); introduced in (Ladevèze, 1997; Ladevèze et al., 2009), RPM has been developed in (Néron and Ladevèze, 2012; Capaldo et al., 2016).

\subsection{The reference point method (RPM)}

Definition of the reference points. - Let us divide the time interval $I=[0, T]$ being studied into $m$ subintervals $\left\{I_{i}\right\}_{i=1, \ldots, m}$ of lengths $\left\{\Delta t_{i}\right\}_{i=1, \ldots, m}$ as shown in Fig. 7. Introducing the centers $\left\{t_{i}\right\}_{i=1, \ldots, m}$ of these subintervals, called "reference times", one has $I_{i}=\left[t_{i}-\Delta t_{i} / 2, t_{i}+\Delta t_{i} / 2\right]$.

In the space domain, let us also introduce $m^{\prime}$ points $\left\{\boldsymbol{x}_{j}\right\}_{j=1, \ldots, m^{\prime}}$ and partition $U$ into $\left\{U_{j}\right\}_{j=1, \ldots, m^{\prime}}$ as shown in Fig. 8. These points are called "reference points" and the measures of the subdomains are denoted $\left\{\omega_{j}\right\}_{i=j, \ldots, m^{\prime}}$. In practice, there would usually be a few dozen reference points.

Let us consider that one needs 20 modes to describe the solution. One can take twice space reference points i.e. 40 and for the reference times the minimum between 40 and the number of time dofs.

Generalized components. - To describe a function defined over $I \times \mathrm{U}$, one consider its generalized components:

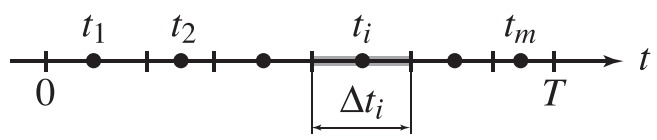

Fig. 7. The reference times over $[0, T]$.

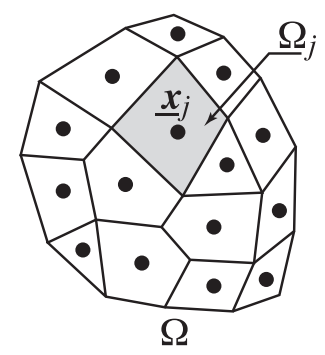

Fig. 8. The reference points over $U$.

$$
\begin{aligned}
\widehat{a}_{i}^{j}(t) & =\left\{\begin{array}{cc}
f\left(t, \boldsymbol{x}_{j}\right) & \text { if } t \in I_{i} \\
0 & \text { otherwise }
\end{array} \text { and } \widehat{b}_{i}^{j}(\boldsymbol{x})\right. \\
& =\left\{\begin{array}{cc}
f\left(t_{i}, \boldsymbol{x}\right) & \text { if } \boldsymbol{x} \in \mathrm{U}_{j} \\
0 & \text { otherwise }
\end{array}\right.
\end{aligned}
$$

with $i=1, \ldots, m$ and $j=1, \ldots, m^{\prime}$. The sets $\left\{\left(\widehat{a}_{i}^{j}, \widehat{b}_{i}^{j}\right)\right\}_{i=1, \ldots, m}^{j=1, \ldots, m^{\prime}}$ are the generalized components of $f$. One should note that these quantities verify the following compatibility conditions: for $i=1, \ldots, m$ and $j=1, \ldots, m^{\prime}$,

$\widehat{a}_{i}^{j}\left(t_{i}\right)=\widehat{b}_{i}^{j}\left(\boldsymbol{x}_{j}\right)$

It could happen that the quantity $f$ is not well represented over the time-space domain. Then, one adds if necessary a PGD description of the complement.

Computation using generalized components. - All basic operations as sum, product, time and space derivations are inexpensive. That is the interest of RPM. Then, the main question is: how can one build or rebuild a field from its components? We choose to define function $f$ from its components using only one product per timespace subdomain $I_{i} \times U_{j}$ :

$f(t, \boldsymbol{x}): a_{i}^{j}(t) b_{i}^{j}(\boldsymbol{x}) \quad \forall(t, \boldsymbol{x}) \in I_{i} \times \bigcup_{j}$

where the sets $\left\{\left(a_{i}^{j}, b_{i}^{j}\right)\right\}_{i=1, \ldots, m}^{j=1, \ldots, m^{\prime}}$ should be defined from the sets $\left\{\left(\widehat{a}_{i}^{j}, \widehat{b}_{i}^{j}\right)\right\}_{i=1, \ldots, m}^{j=1, \ldots, m^{\prime}}$. However, in this case, we let the time domain play a special role because there are many more spatial degrees of freedom than there are time degrees of freedom. Thus, function $f$ is defined by:

$f(t, \boldsymbol{x}): a_{i}(t) b_{i}(\boldsymbol{x}) \quad \forall(t, \boldsymbol{x}) \in I_{i} \times U$

Let us introduce the following scalar products:

$\langle f, g\rangle_{I_{i}}=\int_{I_{i}} f g d t \quad$ and $\langle f, g\rangle_{\cup_{j}}=\int_{\cup_{j}} f g d U$

In order to obtain the sets $\left\{\left(a_{i}, b_{i}\right)\right\}_{i=1, \ldots, m}$, we minimize:

$J\left(a_{i}, b_{i}\right)=\sum_{j=1}^{m^{\prime}}\left[\omega_{j}\left\|\hat{a}_{i}^{j}(t)-a_{i}(t) b_{i}\left(\boldsymbol{x}_{j}\right)\right\|_{I_{i}}^{2}+\Delta t_{i}\left\|\widehat{b}_{i}^{j}(\boldsymbol{x})-a_{i}\left(t_{i}\right) b_{i}(\boldsymbol{x})\right\|_{\mathrm{U}_{j}}^{2}\right]$

which leads to:

$\frac{f(t, \boldsymbol{x}): a_{i}(t) b_{i}(\boldsymbol{x})=\sum_{k=1}^{m^{\prime}} \omega_{k} \widehat{a}_{i}^{k}(t) \widehat{a}_{i}^{k}\left(t_{i}\right)}{\sum_{k=1}^{m^{\prime}} \omega_{k} \widehat{a}_{i}^{k}\left(t_{i}\right) \widehat{a}_{i}^{k}\left(t_{i}\right) \widehat{b}_{i}^{j}(\boldsymbol{x})}$

This gives an inexpensive reconstruction.

Extension to parameter-dependent functions. - The extension to 
parameter-dependent functions is easy. The generalized components are:

$\widehat{a}_{j}^{k}(t)=f\left(t, \boldsymbol{x}_{j}, \boldsymbol{\mu}_{k}\right) \quad$ if $t \in I$

$\widehat{b}_{i}^{k}(M)=f\left(t_{i}, \boldsymbol{x}, \boldsymbol{\mu}_{k}\right) \quad$ if $\boldsymbol{x} \in \Omega$

$\widehat{c}_{i}^{j}(\boldsymbol{\mu})=f\left(t_{i}, \boldsymbol{x}_{j}, \boldsymbol{\mu}\right) \quad$ if $\boldsymbol{\mu} \in \boldsymbol{\Sigma}_{\boldsymbol{\mu}}$

\subsection{Application of RPM}

One considers the model example introduced in Grepl et al. (Grepl et al., 2007) to illustrate the behavior of the EIM approach and used also in Chaturantabut and Sorensen (Chaturantabut and Sorensen, 2010) for the DEIM. This is a parametric nonlinear 2D problem which consists in finding $u(\boldsymbol{x}, \mu)$ with $\left.\boldsymbol{x}=\left(x_{1}, x_{2}\right) \in \Omega=\right] 0,1\left[{ }^{2}\right.$ and $\boldsymbol{\mu}=\left(\mu_{1}, \mu_{2}\right) \in \boldsymbol{\Sigma}_{\boldsymbol{\mu}}=[0.01,10]^{2}$ and homogeneous Dirichlet boundary condition on $\partial \Omega$, such that:

$-\nabla^{2} u+\frac{\mu_{1}}{\mu_{2}}\left(e^{\mu_{2} u}-1\right)=100 \sin \left(2 \pi x_{1}\right) \sin \left(2 \pi x_{2}\right)$

This model can be interpreted as a 2D stationary diffusion problem with a nonlinear interior heat source density. It has the particularity to present strong variations of the solution regarding the values of the parameter $\mu$. The space is discretized through 2500 P1-elements, the parameter set is defined by 225 points i.e. $(15 \times 15)$. Fig. 9 shows the reference solution, denoted $u_{\text {ref, }}$ of the problem for two values of the parameter $\mu$ and one can notice that the studied problem is strongly nonlinear.

To compare the quality of results obtained by a direct approach and with the RPM, the following error is introduced:

$\varepsilon=1 / p \sum_{j=1}^{p} \frac{\left\|u_{r e f}\left(\boldsymbol{x}, \mu_{j}\right)-u_{R P M}\left(\boldsymbol{x}, \mu_{j}\right)\right\|_{L_{2}}}{\left\|u_{r e f}\left(\boldsymbol{x}, \boldsymbol{\mu}_{j}\right)\right\|_{L_{2}}}$

Fig. 10 gives the error in terms of the number of PGD modes for various numbers of reference points. The notation $\left(m_{\mu}, m_{x}\right)$ means that $m_{\mu}$ parameter reference points and $m_{x}$ space reference points. One can see that with very few reference points, one is close to the optimal curve. It appears also still with very few reference points even if one needs more PGD modes, one still converges toward the reference solution. That is a remarkable property.

Fig. 5 shows the gain which is important for the updating stage. However for this academic example, the total gain comparing the PGD alone of the PGD combined to the RPM is about 3. For more complex problems, RPM implementation is in progress and one can expect much higher values (See Table 1).

The interested reader can find in (Capaldo et al., 2016), a comparison of the behavior of the RPM and of the EIM.

\section{A parametrized engineering problem}

To illustrate the use of the technique to deal with parametrized problems, we consider an example issued from (Néron et al., 2015) and which is freely inspired from a blade of the Vulcain engine of

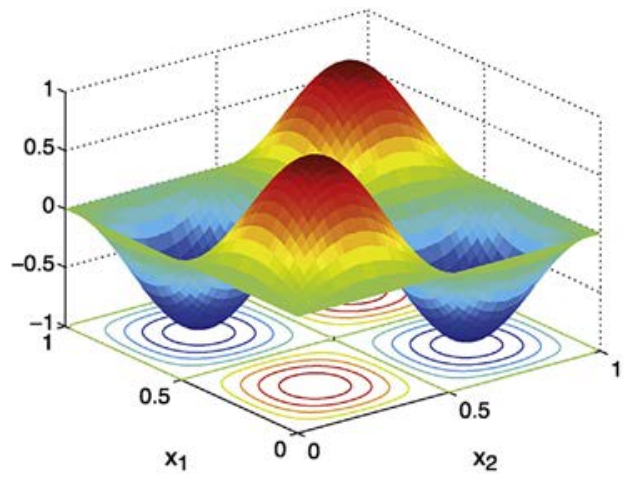

(a) $\boldsymbol{\mu}=(0.010 .01)$

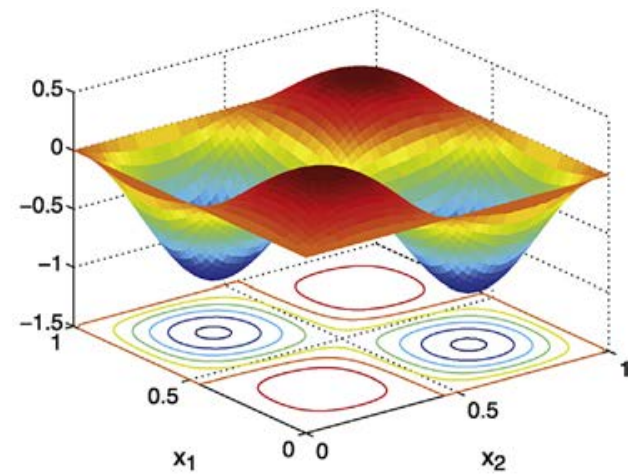

(b) $\boldsymbol{\mu}=(1010)$

Fig. 9. Reference solution $u_{r e f}$ for $\boldsymbol{\mu}=(0.01,0.01)$ and $\boldsymbol{\mu}=(10,10)$.
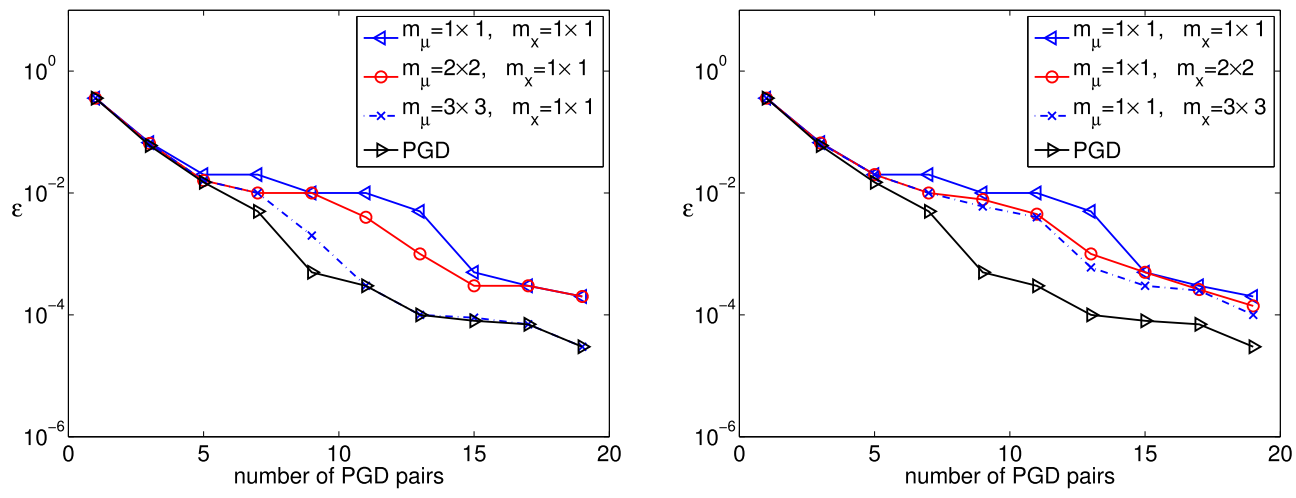

Fig. 10. Error in terms of the number of PGD modes for several reference points. 
Table 1

$\mathrm{CPU}$ time gain with respect to the reference for a given level of error $\left(\varepsilon=10^{-2}\right)$.

\begin{tabular}{|c|c|c|c|c|}
\hline & PGD & PGD-RPM & PGD-RPM & PGD-RPM \\
\hline & & $\overline{n_{\mu}=1 \times 1}$ & $\overline{n_{\mu}=2 \times 2}$ & $n_{\mu}=3 \times 3$ \\
\hline & & $n_{\mu}=1 \times 1$ & $n_{\mu}=1 \times 1$ & $n_{\mu}=1 \times 1$ \\
\hline Number of PGD pairs & 7 & 9 & 7 & 7 \\
\hline Gain with respect to direct method & 6 & 18 & 14.2 & 11.5 \\
\hline
\end{tabular}

the Ariane 5 launcher. The geometry, boundary conditions and mesh are presented on Fig. 11. A four-sinusoidal-cycles displacement with is prescribed on the lower part. The total number of DOFs is 141,500 and the time interval is discretized using 120 time steps. The material coefficients used for the Marquis-Chaboche elastic-viscoplastic material are typical of a Titanium TA6V material at $500^{\circ} \mathrm{K}$.

A first run is carried out and we compare on Fig. 12 the Von Mises fields obtained with ABAQUS and the LATIN method. The comparison shows that there is no appreciable difference between this two fields (the same scale is used for the two plots). A quantitative comparison is presented on Fig. 13: the stress-versus-strain curve is plotted at the most loaded gauss point. Again, the two curves obtained with ABAQUS and the LATIN method are almost identical. Fig. 14 shows the first 3 modes of the PGD decomposition of equivalent plastic strain.
Then, we perform the parametric study of Table 2, which concerns the influence of the loading amplitude, of the limit stress and the power coefficient in the evolution law. The range of variation of each parameter was discretized into 10 values, leading to 1000 different problems.

The chosen three parameters play an important role on the value of the maximum Von Mises stress. Indeed, the virtual chart depicted by Fig. 15 shows the maximum Von Mises stress as a function of the parameters: the variation of this quantity is more than $35 \%$.

To illustrate the performances of the LATIN-PGD with multiple runs algorithm, we compare the CPU times:

- about 50 days (estimated time) are necessary to complete the 1000 resolutions with ABAQUS;

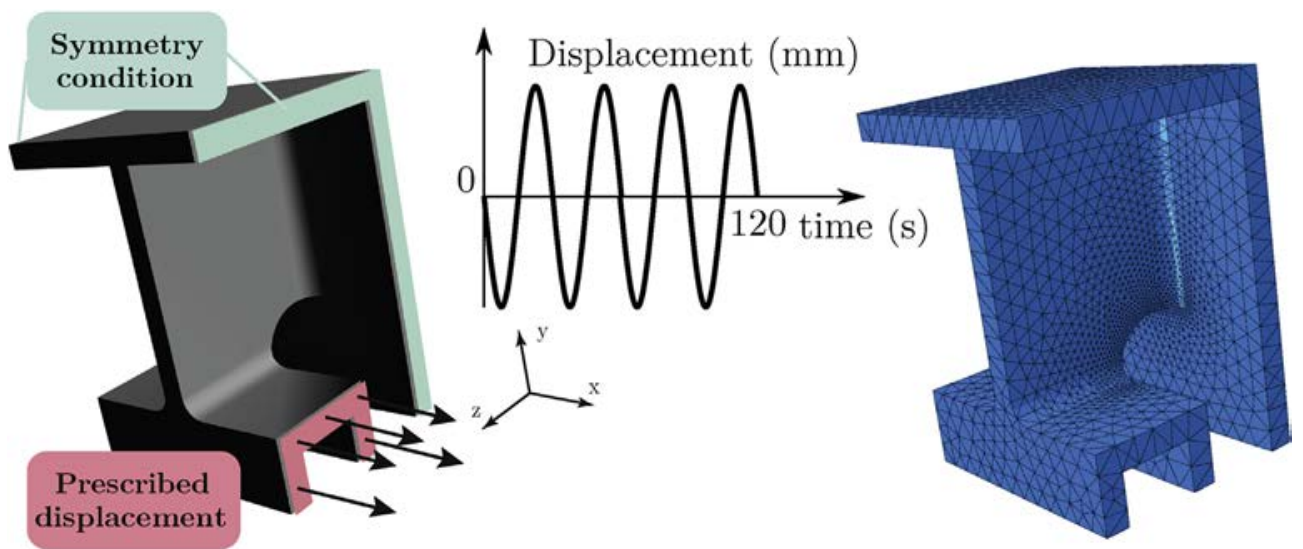

Fig. 11. Geometry, boundary conditions and mesh of the blade test-case.
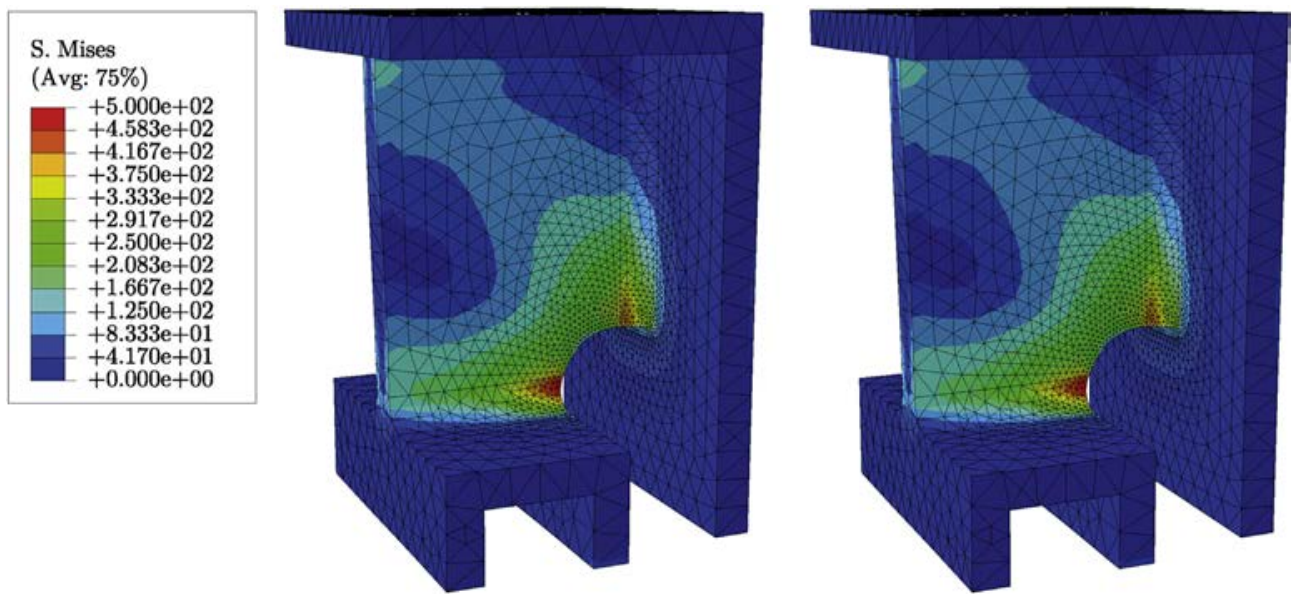

Fig. 12. Comparison of the Von Mises fields obtained at time step 55 with the LATIN (left) and ABAQUS (right). 


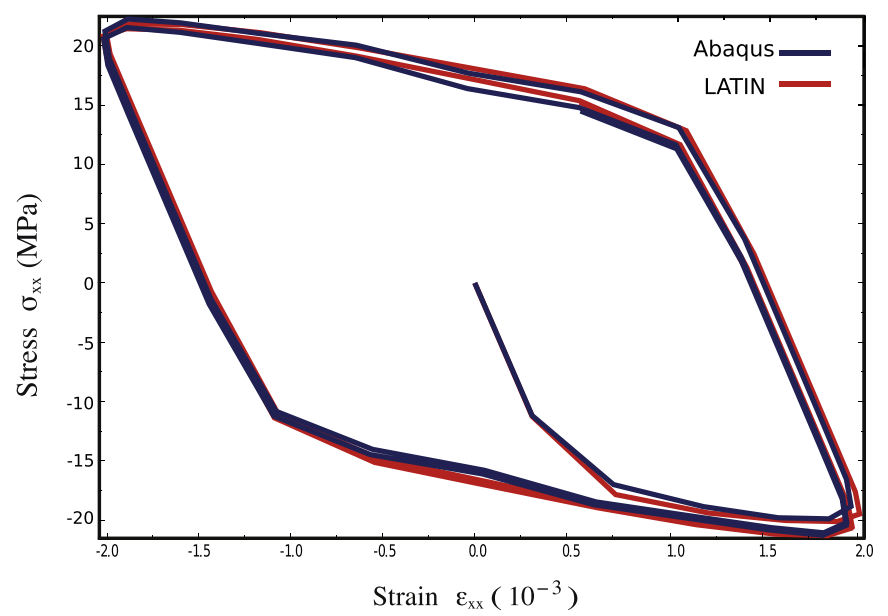

Fig. 13. Stress vs strain curves at the most loaded gauss point.

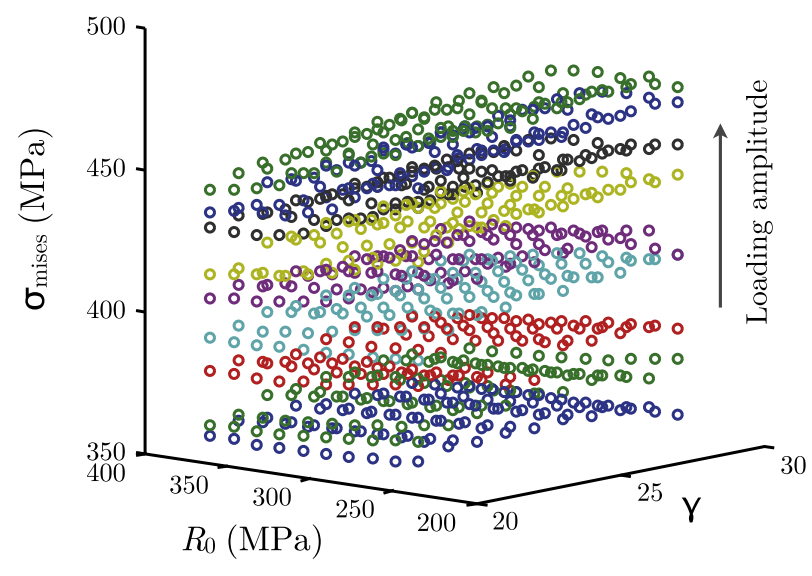

Fig. 15. Maximum Von Mises stress as a function of the parameters.

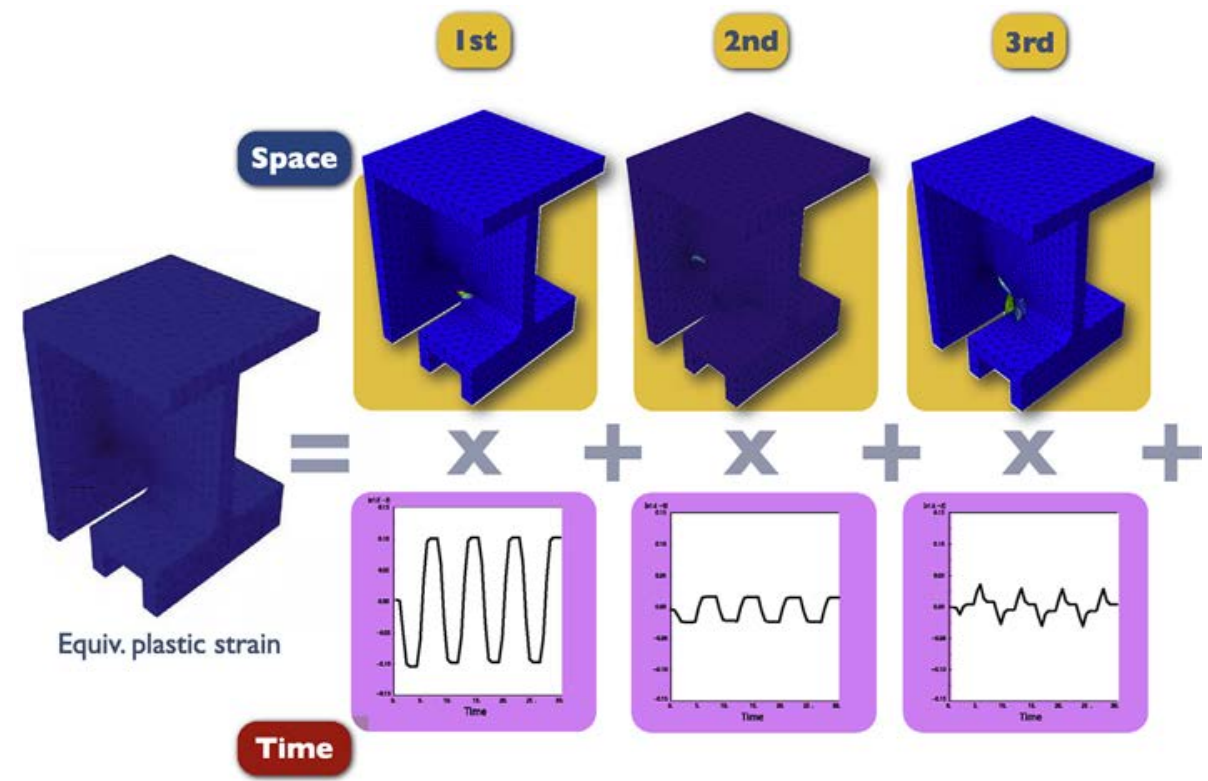

Fig. 14. First 3 modes of the PGD decomposition.

Table 2

Range of variation of loading amplitude, $R_{0}$ and $\gamma$.

\begin{tabular}{lllll}
\hline Parameter & Min. value & Max. value & Step & Range of variation \\
\hline \pm Loading amplitude & $0.1 \mathrm{~mm}$ & $0.19 \mathrm{~mm}$ & $0.01 \mathrm{~mm}$ & $\pm 31 \%$ \\
Limit stress $R_{0}$ & $20 \mathrm{MPa}$ & $29 \mathrm{MPa}$ & $1 \mathrm{MPa}$ & $\pm 18 \%$ \\
Power $\gamma$ & 285 & 330 & 5 & $\pm 7 \%$ \\
\hline
\end{tabular}

- about 18 days (estimated time) are necessary to complete the 1000 resolutions with the classical LATIN-PGD;

- less than $17 \mathrm{~h}$ are necessary to complete the 1000 resolutions with the LATIN-PGD with multiple runs algorithm.

The gain is an about 70 using the multiple runs algorithm, but can achieve more than 700 when using also the RPM strategy. The important point is that, once this parametric study has been performed, a reduced-model of the nonlinear problem is built. For example, some stochastic studies can be performed very easily. Assuming a probabilistic distribution of the three parameters, a probabilistic distribution of the quantity of interest can be computed in quasi-real time.

\section{Conclusion and prospects}

LATIN-PGD with its last version is a robust method to compute ROM for a rather large family of time-dependent nonlinear problems in Solid Mechanics. It includes (visco)-plasticity with moderate damage but for small displacement problems; the number of parameters which could be stochastic variables should be today less than 10. Consequently, engineering applications as the one of paragraph 5 will be more and more numerous. LATIN-PGD has been developed for most of structural mechanics problems but robust ROM computational methods are still missing for several important issues:

- cyclic viscoplasticity (Cognard and Ladevèze, 1993; Cognard et al., 1999);

- large displacement problems with instabilities (Aubard et al., 2002; Boucard et al., 1997; Bussy et al., 1990); 
- damage and final fracture (in particular for composites);

- multiscale and multiphysics problems (Néron and Ladevèze, 2010; Ladevèze and Nouy, 2003; Ladevèze et al., 2007; Nouy and Ladevèze, 2004; Néron and Dureisseix, 2008);

- verification (Ladevèze and Chamoin, 2011; Chamoin and Ladevèze, 2012; Ladevèze and Chamoin, 2012).

For all these issues, the LATIN-PGD version described in this paper should be a paradigm. However, ROM computation is still a scientific challenge for several items as:

\section{- transient dynamics (time/space separation); \\ - very large number of parameters.}

\section{and works are still in progress.}

\section{References}

Allier, P.-E., Chamoin, L., Ladevèze, P., 2015. Proper generalized decomposition computational methods on a benchmark problem: introducing a new strategy based on constitutive relation error minimization. Adv. Model. Simul. Eng. Sci. 2 (1), $1-25$.

Ammar, A., Mokdad, B., Chinesta, F., Keunings, R., 2007. A new family of solvers for some classes of multidimensional partial differential equations encountered in kinetic theory modeling of complex fluids: Part II: transient simulation using space-time separated representations. J. Newt. Fluid Mech. 144 (2-3), 98-121.

Astrid, P. Weiland, S, Willcox, K, Backx, T, 2008. Missing point estimation in models described by proper orthogonal decomposition. IEEE Trans. Autom. Control 53 (10), 2237-2251.

Aubard, X., Boucard, P.-A., Ladevèze, P., Michel, S., 2002. Modeling and simulation of damage in elastomer structures at high strains. Comput. Struct. 80, 2289-2298.

Barrault, M., Maday, Y., Nguyen, N.C., Patera, A.T., 2004. An "empirical interpolation" method: application to efficient reduced-basis discretization of partial differential equations. Comptes Rendus Acad. Des. Sci. Paris 339, 667-672.

Boucard, P.-A., Ladevèze, P., 1999. A multiple solution method for non-linear structural mechanics. Mech. Eng. 50 (5), 317-328.

Boucard, P.-A., Ladevèze, P., Poss, M., Rougée, P., 1997. A nonincremental approach for large displacement problems. Comput. Struct. 64, 499-508.

Bussy, P., Rougée, P., Vauchez, P., 1990. The large time increment method for numerical simulation of metal forming processes. In: NUMETA. Elsevier pp. 102-109.

Capaldo, M., Néron, D., Guidault, P.-A., Ladevèze, P., 2016. An Approximation Framework Dedicated to PGD Based Non-linear Solver. In preparation.

Carlberg, K., Farhat, C., December 2010. A low-cost, goal-oriented 'compact prope orthogonal decomposition' basis for model reduction of static systems. Int. J. Numer. Methods Eng. 86, 341-402.

Chamoin, L., Ladevèze, P., 2012. Robust control of pgd-based numerical simulations Eur. J. Comput. Mech. 21 (3-6), 195-207.

Chaturantabut, S., Sorensen, D., 2010. Nonlinear model reduction via discrete empirical interpolation. Siam J. Sci. Comput. 32 (5), 2737-2764.

Chinesta, F., Ladevèze, P. (Eds.), 2014. Separated Representations and PGD-based Model Reduction. CISM International Centre for Mechanical Sciences. Springer-Verlag Wien.

Chinesta, F., Leygue, A., Bordeu, F., Aguado, J.V., Cueto, E., Gonzalez, D., Alfaro, I. Ammar, A., Huerta, A., 2013. PGD-based computational vademecum for efficien design, optimization and control. Arch. Comput. Methods Eng. 20 (1), 31-59.

Cognard, J.-Y., Ladevèze, P., 1993. A large time increment approach for cyclic viscoplasticity. Int. J. Plast. 9, 141-157.

Cognard, J., Ladeveze, P., Talbot, P., Sep-Nov 1999. A large time increment approach for thermo-mechanical problems. Adv. Eng. Softw. 30 (9-11), 583-593.

Courard, A., Néron, D., Ladevèze, P., 2016. A new pgd approach for problems including positional parameters. to appear Adv. Model. Simul. Eng. Sci.

Everson, R., Sirovich, L., March 1994. Karhunen-loève procedure for gappy data. J. Opt. Soc. Am. 12 (8), 1657-1664.

Golub, G.H., Van Loan, C.F., 1996. Matrix Computations, 3nd edn. Johns Hopkins University Press, Baltimore.

Grepl, M., Maday, Y., Nguyen, N.C., Patera, A., 2007. Efficient reduced-basis treatment of nonaffine and nonlinear partial differential equations. ESAIM Math Model. Numer. Anal. 41, 575-605.

Gunzburger, M., Peterson, J., Shadid, J., 2007. Reduced-order modeling of timedependent PDEs with multiple parameters in the boundary data. Comput. Methods Appl. Mech. Eng. 196, 1030-1047.

Hernández, J.A., Oliver, J., Huespe, A.E., Caicedo, M.A., Cante, J.C., 2014. High-performance model reduction techniques in computational multiscale homogenization. Comput. Methods Appl. Mech. Eng. 276 (0), 149-189.

Heyberger, Ch, Boucard, P.-A., Néron, D., 2013. A rational strategy for the resolution of parametrized problems in the PGD framework. Comput. Methods Appl. Mech. Eng. 259, 40-49.

Jessus, M., Néron, D., Ladevèze, P., 2016. Low-cost PGD-reduced Models in Nonlinear
Solid Mechanics Including Cyclic Loadings. In preparation.

Kunisch, K. Xie, L., 2005. Pod-based feedback control of the burgers equation by solving the evolutionary hjb equation. Comput. Math. Appl. 49 (7-8), $1113-1126$.

Ladevèze, P., 1985. New Algorithms: Mechanical Framework and Development (In French). Technical Report 57. LMT-Cachan.

Ladevèze, P., 1985. On a family of algorithms for structural mechanics (in french). Compte rendu l'acad. Sci. 300 (2), 41-44.

Ladevèze, P., 1989. The large time increment method for the analyse of structures with nonlinear constitutive relation described by internal variables. Comptes Rendus Acad. Des. Sci. Paris 309 (II), 1095-1099.

Ladevèze, P., 1997. A Computational Technique for the Integrals over the Time-space Domain in Connection with the LATIN Method (In French). Technical Report 193. LMT-Cachan.

Ladevèze, P., 1999. Nonlinear Computational Structural Mechanics-new Approaches and Non-incremental Methods of Calculation. Springer Verlag.

Ladevèze, P., 2008. Strict upper error bounds for computed outputs of interest in computational structural mechanics. Comput. Mech. 42 (2), 271-286.

Ladevèze, P., Chamoin, L., 2011. On the verification of model reduction methods based on the proper generalized decomposition. Comput. Methods Appl. Mech. Eng. 200, 2032-2047.

Ladevèze, P., Nouy, A., 2003. On a multiscale computational strategy with time and space homogenization for structural mechanics. Comput. Methods Appl. Mech. Eng. 192, 3061-3087.

Ladevèze, P., Perego, U., 2000. Duality preserving discretization of the large time increment methods. Comput. Methods Appl. Mech. Eng. 189 (1), 205-232.

Ladevèze, P., 1991. New advances in the large time increment method. In: Ladevèze, P., Zienkiewicz, O.C. (Eds.), New Advances in Computational Structural Mechanics. Elsevier, pp. 3-21.

Ladevèze, P., Néron, D., Gosselet, P., 2007. On a mixed and multiscale domain decomposition method. Comput. Methods Appl. Mech. Eng. 196, 1526-1540.

Ladevèze, P., Néron, D., Passieux, J.-C., 2009. On multiscale computational mechanics with time-space homogenization. In: Fish, J. (Ed.), Multiscale Methods-Bridging the Scales in Science and Engineering, Chapter Space Time Scale Bridging Methods. Oxford University Press, pp. 247-282.

Ladevèze, P., Passieux, J.-C., Néron, D., 2010. The LATIN multiscale computational method and the Proper Generalized Decomposition. Comput. Methods Appl. Mech. Eng. 199, 1287-1296.

Ladevèze, P., Chamoin, L., 2012. Toward guaranteed PGD-reduced models. In: Zavarise, G., Boso, D. (Eds.), Bytes and Science. CIMNE, Barcelona, Spain.

Lamari, H., Ammar, A., Cartraud, P., Legrain, G., Chinesta, F., Jacquemin, F., 2010 Routes for efficient computational homogenization of nonlinear materials using the proper generalized decompositions. Arch. Comput. Methods Eng. 17, 373-391.

Lieu, T., Farhat, C., Lesoinne, A., 2006. Reduced-order fluid/structure modeling of a complete aircraft configuration. Comput. Methods Appl. Mech. Eng. 195 (41-43), 5730-5742.

Maday, Y., Ronquist, E.M., 2004. The reduced-basis element method: application to a thermal fin problem. J. Sci. Comput. 26 (1), 240-258.

Néron, D., Dureisseix, D., 2008. A computational strategy for thermo-poroelastic structures with a time-space interface coupling. Int. J. Numer. Methods Eng. 75 (9), 1053-1084.

Néron, D., Ladevèze, P., 2010. Proper Generalized Decomposition for multiscale and multiphysics problems. Arch. Comput. Methods Eng. 17 (4), 351-372.

Néron, D., Ladevèze, P., 2012. A data compression approach for PGD reduced-order modeling. In: Proceedings of the 11th Biennial Conference on Engineering Systems Design and Analysis. ASME.

Néron, D., Boucard, P.-A., Relun, N., 2015. Time-space PGD for the rapid solution of 3D nonlinear parametrized problems in the many-query context. Int. J. Numer. Methods Eng. 103 (4), 275-292.

Nouy, A., Ladevèze, P., 2004. Multiscale computational strategy with time and space homogenization: a radial type approximation technique for solving micro problems. Int. J. Multiscale Comput. Eng. 170 (2), 557-574.

Patera, A., Rozza, G., 2006. Reduced Basis Approximation and a Posteriori Error Estimation for Parametrized Partial Differential Equations. Version 1.0. MIT.

Radermacher, A., Reese, S., 2015. Pod-based model reduction with empirical interpolation applied to nonlinear elasticity. Int. J. Numer. Methods Eng. http:// dx.doi.org/10.1002/nme.5177.

Relun, N., Néron, D., Boucard, P.-A., 2013. A model reduction technique based on the PGD for elastic-viscoplastic computational analysis. Comput. Mech. 51 (1), 83-92.

Rozza, G., Veroy, K., 2007. On the stability of the reduced basis method for Stokes equations in parametrized domains. Comput. Methods Appl. Mech. Eng. 196 (7), 1244-1260.

Ryckelynck, D., 2005. A priori hyperreduction method: an adaptive approach. J. Comput. Phys, 202, 346-366.

Ryckelynck, D., 2009. Hyper-reduction of mechanical models involving internal variables. Int. J. Numer. Methods Eng. 77 (1), 75-89.

Ryckelynck, D., Vincent, F., Cantournet, S., 2012. Multidimensional a priori hyperreduction of mechanical models involving internal variables. Comput Methods Appl. Mech. Eng. 225â228, 28-43.

Yvonnet, J., Monteiro, E., He, Q.C., 2013. Computational homogenization method and reduced database model for hyperelastic heterogeneous structures. Int. J. Multiscale Comput. Eng. 11 (3), 201-225. 